\title{
Iatrogenic common bile duct injuries: Intra-operative identification and management
}

\author{
Phillip Jeans, MBBS, FRACS \\ Consultant Hepatobiliary Surgeon, The Canberra Hospital, Australia
}

\begin{abstract}
Key Words: Bile duct injuries, Identifying injuries, Cholangiography
\end{abstract}

\section{Introduction}

Prior to the introduction of laparoscopic cholecystectomy in 1987 the main rule for avoiding bile duct injuries was to expose the Common Bile Duct (CBD)/ Cystic Duct junction clearly but despite this bile duct injuries still occurred at a fairly predictable rate.

Laparoscopic Cholecystectomy changed our perceptions of the anatomy and the approach to the operation. The main change in our surgical approach was to stress the importance of identifying the gallbladder neck/cystic duct junction and to try and keep away from the cystic duct/CBD junction but still bile duct injuries occur at a frustrating rate of 1 in every 300-400 cases. Many authors have described the injuries and their management $[1,2]$ and proposed systems of classification [3] but the two most used systems of classification are the older but simple Bismuth classification or the more precise Strasberg classification [4].

\section{How can we recognise these injuries?}

The first important thing to recognise is that there are many variations in anatomy and other factors such as obesity and pathological distortion which will compound in obscuring the true anatomy. There are several variations which are hard to recognise and the one which is most difficult to identify is drainage of the gallbladder directly into a right hepatic, right sectoral or segmental duct with possible absence of

Correspondence: Phillip Jeans, Consultant Hepatobiliary

Surgeon, The Canberra Hospital, Australia

Email: p.jeans@gastrotract.com.au

The Sri Lanka Journal of Surgery 2011; 29(2):84-86 the cystic duct. In this situation the right duct will look like it is the cystic duct coming from the neck of the gallbladder [5].

Injuries are potentially far worse when combined with a vascular injury [6].

\section{Perform Cholangiography}

Injuries to the ducts (particularly the cystic duct) can occur during cannulation for cholangiography but these injuries will usually be less serious than what might occur without a cholangiogram.

a. The Reddick-Olsen cholangiogram forceps can obtain a cholangiogram with a minimal cannulation so the catheter doesn't have to be forced further than it is comfortable going. This will prevent catheter perforations of the cystic duct from forceful cannulation. I believe it is a superior technique than other available techniques. Cholangiography can also be performed through the neck of the gallbladder if there is doubt about the anatomy.

b. Try to get filling of the common hepatic duct and intrahepatic ducts. If you see an anomaly, be warned, expect more anomalies and take care. (In my own case of absent cystic duct draining into a right sectoral duct, the segment III an IVB ducts drained into the other right sectoral duct and there was a rare anomaly of the hepatic venous anatomy.)

If you can't see the common hepatic duct consider manoeuvres to enhance the filling proximal to the catheter. Sometimes just moving the tip of the cholangiogram forceps during the cholangiogram (by levering the handle) will allow the proximal ducts to fill. Consider tipping the patient head down. Use a 
big enough catheter and an appropriate sized syringe to get rapid volumes of contrast into the duct. Speed of delivery is quicker with a $20 \mathrm{ml}$ syringe than a $30 \mathrm{ml}$ syringe. A $10 \mathrm{ml}$ syringe would generate even higher pressure but the volume may be inadequate.

c. If we have made a mistake in identification then we should recognise that we have a side hole in the CBD or other duct before we make it worse by dividing it.

\section{Recognising an Injury}

\section{The different scenarios}

\section{Case 1. Intraoperative recognition of a duct injury by cholangiogram}

Step 1. The cholangiogram is the often the first hint that an injury has occurred; e.g. it is apparent that the wrong duct has been cannulated.

Step 2. If this is the case, reposition the catheter in the duct to take a cholangiogram in the reverse direction to decide the anatomy. Decide the seriousness of the injury and how it might be managed.

Step 3. Ask yourself whether you are the right person to be fixing it? Even if you have the training and skill to do the appropriate operation to minimise damage or correct the problem are you calm enough to do it well? (Most of us are not calm in this situation and that clouds our thinking.) Is there a colleague nearby to help the decision making process and the surgery? Would it be better if they did the operation and you assisted them?

Step 4. If you don't satisfy these criteria then consider putting in a drain and transferring the patient to a facility that can deal with the problem.

\section{Case 2. You encounter an "extra" duct}

This is a very deflating experience for a surgeon. If it has already been cut and is draining bile...perform a cholangiogram into it. This will help to define the problem. If it is not cut, do no further damage. If you can't discern the anatomical mistake call for help or back off and transfer the patient to a HPB unit to deal with it. If it is a duct of Lushka in the gallbladder bed it can be managed with clipping or oversewing but leave a drain but beware....in the case of the rare anatomical variations, significant segmental and sectoral ducts can enter the liver well up in the gallbladder bed. Small segmental ducts can often be left to drain bile and sclerose or be clipped off. If it can be clipped off leave a drain as the clip can cut through an obstructed duct and leak bile. Surgical opinion is divided about the outcome of damaged segmental ducts. Many recover without any problem though some cases can develop pain which is only relieved by performing the appropriate segmentectomy.

\section{Case 3. You have just divided the CBD and you recognise it}

This is a situation which does allow reanastomosis at the same operation as long as there is no loss of length and as long as there has not been a vascular injury with it. It is preferably closed over a T-tube to allow stenting and further cholangiographic access.

\section{Case 4. You clip the CBD and recognise it}

I have seen this twice and on each occasion the outcome was vastly different. In one there was no problem but the other presented with a tight stricture one month later that couldn't be wired for stenting at both ERCP and PTC and this case required open surgery. If there appears to be a crush injury with a dark line where the clip has been, then insertion of a $\mathrm{T}$ tube is a wise manoeuvre, otherwise transfer the patient for early endoscopic stenting.

\section{Case 5. You have clipped or tied part of the CBD by tenting the cystic duct too much}

If you recognise this injury at the time then the best approach is to remove the suture or clips and that may leave you with damage to one side of the $\mathrm{CBD}$; excise that and put a t-tube in the opening. This type of injury can heal without stricturing if it is stented with a t-tube 
in the opening. This type of injury can heal without stricturing if it is stented with a t-tube for a while. Later the t-tube can be replaced with an endoscopic stent if there is a lingering concern.

\section{Case 6. You have removed the gallbladder and on inspecting it you see there is more than just a cystic duct attached to it}

It is not too late to re-establish the pneumoperitoneum and have a look....see if you can see an open duct and do cholangiography into it or leave a drain.

\section{Conclusion}

These are the cases that are recognised at the time of surgery. Sometimes we are clever enough to recognise an anatomical variation before cutting it or incising it but often in this situation the dissection has skeletonised the duct too much, which devascularises it and it necroses or scleroses later. Many bile duct injuries present later because of a bile leak, jaundice, pain or abnormal LFTs. They require assessment and management in specialised units.

In Western societies bile duct injuries are regarded as unwinnable legal cases but the constancy of the rate of injury suggests that this is a problem inherent in biliary surgery and should not be regarded as necessarily negligent. The main difference between good units and bad units is the degree of injury and the timeliness of recognition. Some of these injuries occur because of perception problems that occur with repetitive procedures and some occur due to the nasty pathology encountered. The most important thing is to act responsibly and get help. If the appropriate help is not close at hand transfer the patient to an institution where it is.

\section{References}

1. Shallaly GEI and Cuschieri A. Nature, aetiology and outcome of bile duct injuries after laparoscopic cholecystectomy. HPB 2000:2;3-12.

2. McPartland KJ and Pomposelli JJ. Iatrogenic biliary injuries: Classification, identification and Management. Surg Clin N Am 2008:88;1329-1343.

3. Michek J, Zelnicek P, Cchmann J, Svoboda P, Vrastyak J, Cierny M and Kozumplik L. New Classification of major bile duct injuries associated with laparoscopic cholecystectomy. Scripta Medica (Brno) 2002:75;283-290.

4. Strasberg SM, Hertl M and Soper NJ. An analysis of the problem of biliary injury during laparoscopic cholecystectomy. J Am Coll Surg 1995:180;101-125.

5. Prinz RA, Slade Howell $\mathrm{H}$ and Pickleman JR. Surgical significance of extrahepatic biliary tree anomalies. Am J Surg 1976:131;755-757.

6. Strasberg SM and Helton WS. An analytical review of vasculobiliary injury in laparoscopic and open cholecystectomy. HPB 2011:13;1-14. 